\title{
Prudent Creditization Polling (PCP): A novel adaptive polling service for an EPON
}

\author{
Burak Kantarcı, Mehmet Tahir Sandıkkaya, Ayşegül Gençata, and Sema Oktuğ \\ Istanbul Technical University, \\ Department of Computer Engineering, \\ Computer Networks Research Laboratory \\ 34469 Maslak, Istanbul, Turkey \\ \{bkantarci, sandikkaya, \\ gencata, oktug\}@itu.edu.tr \\ http://www.ce.itu.edu.tr
}

\begin{abstract}
In this paper a novel adaptive bandwidth allocation scheme called Prudent Creditization Polling (PCP) is proposed and adapted with the reservation protocol IPACT. PCP attempts to reduce the average queue length at the ONUs and the average packet delay throughout the network. At each polling period, PCP attempts to creditize the request of the ONUs that suffer from high RTT, high average delay per packet, and high buffer growth. We implemented PCP with a fuzzy functional approach and a linear regression approach. We simulated PCP and several service disciplines in IPACT and compared their performance in terms of average packet delay and queue length. We show that PCP improves the performance of IPACT significantly in terms of the performance metrics aforementioned.
\end{abstract}

Key words: Optical Access Network, Passive Optical Network, EPON, IPACT, Multipoint Control Protocol.

\section{Introduction}

Although deployment of optical technology in the backbone has provided sufficient solutions, the bottleneck between the high capacity local area network and the backbone seems to suffer from bandwidth problems. Hence, Passive Optical Networks (PONs) are being thought as an attractive solution to this, so-called first-mile problem [1].

A PON is a point-to-multipoint optical network with no active elements in the path between source and destination. The data transmission in a PON is performed between the optical line terminal (OLT) and a number of optical network units (ONUs). OLT is the unit by which the access network is connected to the backbone. End users directly get service from the ONU [2]. EPON seems to be the most attractive PON type for the next generation access networks among several PON standards [3].

Since an OLT does not share the transmission media with any other device, there is no possibility of contention in downstream transmission. The challenging 
problem in communication through PON is in the upstream data transmission where a number of ONUs have to share one fiber link to send their frames to the OLT. When more than one ONU attempt to transmit data simultaneously to the OLT, their corresponding frames may collide. The ONUs cannot be aware of each other [3].

Multi-Point Control Protocol (MPCP- IEEE 802.3ah) solves the contention problem in the upstream transmission by the REPORT and GATE messages [3]. Upon registering the network, each ONU generates REPORT messages in which its bandwidth requirement is included. Based on the REPORT messages collected from the ONUs, the OLT grants distinct transmission timeslots to the ONUs by the GATE messages.

Several bandwidth allocation algorithms are proposed to assign non-overlapping timeslots to the ONUs so that each ONU can transmit its frames without a contention although it is not aware of any other ONU in the EPON. A popular scheme is Interleaved Polling with Adaptive Cycle Time (IPACT) [4] which offers a number of service disciplines to assure high throughput and low packet delay throughout the EPON. Besides these, there are also several dynamic bandwidth allocation schemes that attempt to enhance QoS by adapting with DiffServ as proposed in [5-7]. A weighted fair queueing model is proposed in [8] that considers the QoS requirements of different traffic types, and schedules the packet traffic in the same ONU based on their urgency. In [9] each ONU employs a database to keep the granted bandwidths to the other ONUs so that the IPACT scheme is implemented in a decentralized manner. In [10], the authors propose a bandwidth allocation scheme where the excess bandwidth of the lightly loaded ONUs is shared among the heavily loaded ONUs, and the modified IPACT leads to a slight decrease in delay. In [11] a dynamic bandwidth allocation scheme based on analytical linear regression of the ONU buffer size is proposed.

In the IPACT scheme, OLT keeps track of $T_{\text {schedule }}$, the earliest scheduling time, on the upstream link. Whenever OLT allocates a new timeslot for an ONU, the value of $T_{\text {schedule }}$ is updated, and whenever a REPORT message containing an ONU's bandwidth request arrives at the OLT, the OLT provides a guard time just after the previously reserved timeslot, and allocates the start of the ONU's transmission window. Based on the service disciplines employed, the OLT grants a timeslot for the ONU, and using the length of this timeslot $(L)$, it immediately updates $T_{\text {schedule }}$ for the following REPORT messages. These service disciplines can be limited service, TDMA (fixed service), constant credit service, gated service, linear credit service, elastic service schemes [4]. The previous research on this subject $[4,9,10]$ shows that all of the service schemes coincide in terms of average delay per packet and average queue length under heavy traffic load.

In this paper, we propose a novel adaptive polling algorithm called Prudent Creditization Polling (PCP) that attempts to decrease average packet delay and average queue size. Based on the decrease in these parameters; the overall packet loss rate due to buffer overflow decreases. As we define in the following sections, PCP adapts the polling cycle time based on the creditization of the ONUs. The request of an $\mathrm{ONU}$ is creditized based on its running average buffer size 
(or its differential), average delay it is exposed to, and its RTT value which is computed at the previous transmission period. Hence, the algorithm aims to decrease the average packet delay by supplying longer transmission window to the ONUs which are exposed to higher delays and faster running average buffer growth. The creditization of the ONUs based on these parameters are done by either a merit based fuzzy functional approach (PCP-Merit) or an analytical linear regression approach (PCP-Regression). We adapt PCP to the IPACT and compare its performance with several service disciplines in IPACT by simulation. The simulation results show that PCP brings a significant improvement on the performance of IPACT in terms of average packet delay and average queue size at high loads.

The paper is organized as follows: The two models of the proposed polling scheme and its adaptation to IPACT is described in detail in Section II. The simulation environment and the results obtained are presented in Section III. Finally, Section IV concludes the paper by giving future considerations.

\section{Prudent Creditization Polling (PCP)}

The first reason of high packet delay and the large amount of queue size is the round-trip times (RTTs) of the ONUs. The signaling between the OLT and the ONU, (including the REPORT and GATE messages) and the packet transmission in the assigned timeslot lead to a buffering delay for the packets that cannot be transmitted at the corresponding timeslot. Another reason for packet delay is the fast growth of the ONU buffer. Hence, the higher running average number of queued packets leads to the higher delay per packet. Besides these, as the packets of an ONU are exposed to a queueing delay, the running average packet delay increases at the ONU. The running average packet delay, in turn, increases the average delay per packet in the EPON. In the former service schemes, the OLT attempts to creditize or weight the ONU requests just based on their instant buffer sizes. However, it does not provision the change of the buffer size in time. Besides this, the OLT considers neither the effect of current request nor buffer growth characteristics, nor the effect of RTT on the weight of the ONU request. Thus, a service scheme that considers the joint effect of these three factors is emergent for the IPACT scheme.

We derive two models to implement the proposed scheme. The first model is based on an analytical linear regression approach (PCP-Regression). The second model is based on a fuzzy functional merit based approach (PCP-Merit).

\subsection{PCP-Regression}

In the PCP-Regression scheme, the difference between the requested and the granted window size is predicted by using an analytical linear regression. We use two previous values of round trip time, buffer size difference and running average packet delay in regression. Modeling buffer size difference $(\delta B S)$ as shown in (1) it is possible to determine the values of $\alpha(3)$ and $\beta$ (4) using well-known least 
squares method which produces the equation set in (2). Using the coefficients, difference between the requested and the granted window size can be calculated as in (5).

$$
\begin{gathered}
\delta B S_{i}(t)=\alpha \cdot R A P D_{i}(t-1)+\beta \cdot R T T_{i}(t-1) \\
{\left[\begin{array}{cc}
\sum R T T_{i}^{2} & \sum R A P D_{i} \cdot R T T_{i} \\
\sum R A P D_{i} \cdot R T T_{i} & \sum R A P D_{i}^{2}
\end{array}\right] \times\left[\begin{array}{c}
\alpha \\
\beta
\end{array}\right]=\left[\begin{array}{c}
\sum \delta B S_{i} \cdot R T T_{i} \\
\sum \delta B S_{i} \cdot R A P D_{i}
\end{array}\right]} \\
\alpha=\frac{\delta B S_{i}(t-1) \cdot R A P D_{i}(t)-\delta B S_{i}(t) \cdot R A P D_{i}(t-1)}{R T T_{i}(t-1) \cdot R A P D_{i}(t)-R T T_{i}(t) \cdot R A P D_{i}(t-1)} \\
\beta=\frac{\delta B S_{i}(t-1) \cdot R T T_{i}(t)-\delta B S_{i}(t) \cdot R T T_{i}(t-1)}{R A P D_{i}(t-1) \cdot R T T_{i}(t)-R A P D_{i}(t) \cdot R T T_{i}(t-1)} \\
\delta B S_{i}(t+1)=\alpha \cdot R A P D_{i}(t)+\beta \cdot R T T_{i}(t)
\end{gathered}
$$

If this result is negative, OLT grants the ONU with the requested window size and if it is greater than zero, OLT grants the ONU by adding this recently calculated value to the requested window size as shown in (6).

$$
\operatorname{GRANT}_{i}(t+1)=R E Q_{i}(i)+\delta B S_{i}(t+1)
$$

The above computation expects the growth with respect to time in the buffers of the ONUs by featuring linear regression. Using linear regression OLT is capable of estimating the future tendency of ONUs and reserve a more suitable transmission window than the ONUs requested. A suitable transmission window is created by determining whether the specified ONU needs a longer transmission window than it requested, or not. If the OLT determines that the tendency is at the negative side, it does not reserve additional time for the ONU. If it determines that the tendency is at positive side, it reserves additional time for the ONU. To keep the system stable, the OLT normalizes the total bandwidth grant. Although, this approach needs a bit more computation than the following one, its time complexity is still $\mathrm{O}(\mathrm{N})$.

\section{$2.2 \quad$ PCP-Merit}

When we construct our model, we consider three factors stated above as the reasons that increase the queuing delay and average delay per packet. Hence, we force OLT to supply more credit to the ONUs which are more likely to be affected by queuing delay. Running average packet delay (RAPD) is computed locally at each ONU just before sending the REPORT message. RAPD is used to calculate the Prudent Creditization (PC) which will later be used to send the state of the ONU to the OLT. This parameter can be computed by (7) where $R A P D_{i}(t)$ stands for the running average packet delay computed at the $O N U_{i}$ at each burstification time $t$. 


$$
R A P D_{i}(t)=\frac{\text { AveragePacketDelay }}{\text { AverageBufferSize }}
$$

When calculating the RAPD parameter, the parameters in the numerator and the denominator are measured continuously. For example, if $B$ bytes arrive at time $t$ to the $O N U_{i}$, the AverageBufferSize is measured as shown below where $\alpha=0.8$. AveragePacketDelay is measured in a similar way;

$$
\text { AverageBufferSize }_{\text {new }}=\text { AverageBufferSize }_{\text {old }} \cdot \alpha+(1-\alpha) \cdot B
$$

Moreover to compute the collective effect of the average packet delay, RTT, and the current buffer size (currently requested bytes) on the throughput of an $\mathrm{ONU}$ is also used. We call this parameter prudent creditization (PC) product. For the $O N U_{i}$ at discrete time $t$ we represent it by $P C_{i}(t)$, and compute it as shown in (8). Here, $R T T_{i}(t)$ is the round trip time that is computed by $O N U_{i}$ at time $t$, and BufferSize $(t)$ is the queue size of the ONU at time $t . P C_{i}(t)$ is similar to a fuzzy-AND function [12] of these three parameters although it does not take values in $[0,1]$. Therefore, based on an analogy to a fuzzy-AND function, $P C_{i}(t+1)$ can be interpreted as "increase the creditization of the ONU whose running average packet delay AND round trip time AND instant buffer size are high enough".

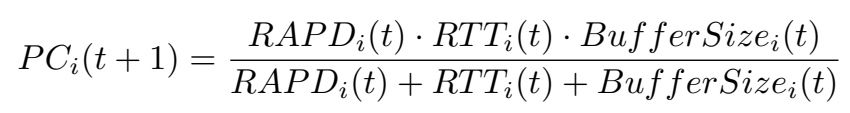

The computed $P C_{i}(t+1)$ value can be transmitted in 4 bytes by padding it into the REPORT message.

The OLT keeps a table for the state of the network where each row of the table represents the metrics computed or collected from the ONUs, namely RTT, requested window size, and $\mathrm{PC}$ product. Upon receiving the REPORT message from an ONU, the OLT updates $P C_{i}(t)$ and derives a normalized value $(\nabla \mathrm{PC})$ with respect to the sum of the current PC product values of all ONUs as given in (3) where $N$ is the total number of ONUs. Normalization provides fairness among the ONUs by avoiding the possibility of fiber monopolization by an ONU with a large RTT, high packet delay and high buffer growth. Therefore $\nabla$ PC takes values in $[0,1]$.

$$
\nabla P C_{i}(t+1)=\frac{P C_{i}}{\sum_{k=1}^{N} P C_{k}}
$$

The request of the $i^{t h} \mathrm{ONU}$ for the $(t+1)^{t h}$ cycle is granted by incrementing it proportional to the normalized value of the prudent creditization factor, as shown in (9) where $G R A N T_{i}(t)$ is the transmission window size responsed by OLT for $O N U_{i}$ and $R E Q_{i}(t)$ stands for the requested transmission windows size by the $i^{\text {th }} \mathrm{ONU}$ at its transmission in the end of $t^{t h}$ cycle.

$$
\operatorname{GRANT}_{i}(t+1)=R E Q_{i}(t+1)+R E Q_{i}(t+1) \cdot \nabla P C_{i}(t+1)
$$


The above computation flow can be summarized as follows: Upon updating the GRANT, the RTT value for the corresponding ONU is re-calculated by the OLT. When calculating the GRANT, the OLT considers the RTT, running average delay, and buffer size. Those considered factors, are combined with the same factors of the other ONUs, and a variable credit is obtained. That variable credit is added to the requested window. This approach is expected to decrease the overall packet delay and queue length in the network. Since in the PON, the packet loss is due to the buffer overflow, as a result of decrease in the average queue length, the packet loss ratio is also expected to decrease. We support this inference in Section IV by the simulation results under different traffic types.

The time complexity of calculating $\nabla P C_{i}(t+1)$ is $\mathrm{O}(\mathrm{N})$. This shows that our scheme does not cause high computational complexity at the OLT. The cost of this technique is an additional $4 \cdot N$ bytes in the state table kept at the OLT to keep the PC product values. In the network traffic point of view, it brings an additional 4 bytes of control information padded to the REPORT message which corresponds to 32 ns at 1 Gbps line rate. These analysis show that PCP-Merit is also cost effective for implementation.

\section{Simulation Study}

\subsection{Simulation Environment}

The 16-node tree topology, given in [4], is used in our simulation scenarios. We assume that the line rate between the users and the ONUs $\left(R_{D}\right)$ is $100 \mathrm{Mbps}$ while the fiber between the ONUs and the OLT has the capacity of 1 Gbps.

We generate two different traffic types at the ONUs: Self-similar traffic with Hurst parameter 0.8, and Poisson traffic. Self-similar traffic is generated as defined in [13] by aggregating 256 sub-streams on $100 \mathrm{Mbps}$ line rate.

The incoming packet size is distributed uniformly in $[64,1500]$ bytes. We generate 1000000 packet traces at each ONU.

The REPORT and GRANT messages are 84 bytes long. We employ a $5 \mu \mathrm{s}$ guard time between two adjacent transmission windows. At the beginning of the simulation, in order to construct the initial state table at the OLT, the RTTs of the ONUs are selected uniformly from the interval $[100,200] \mu s$. At the end of each transmission cycle, the dynamic values of the RTTs are re-calculated. In order to observe the difference between behavior of the services in terms of packet delay and queue length, we set the maximum buffer size to a large value of 1 Gbytes (to introduce delay to all the buffered packets).

We compare the performance of PCP with four different service schemes in IPACT, namely limited, fixed, constant credit, and linear credit services. In limited service scheme we set the $W_{\max }$ to be equal to 15000 bytes, where in fixed service each transmission window is adjusted to transmit 10000 bytes. In constant credit service discipline we add a 1000 bytes credit to the requested window size while we add $\% 0.1$ of the requested window size to the incoming request in order to employ linear credit service scheme (since the traffic is bursty, this ratio 
is observed to be sufficient to be added). Each point in the figures represents the average of ten runs.

\subsection{Simulation Results}

Our simulation results are two-fold: Average packet delay and Average queue length. First, we take the results under long range dependent traffic $(H=0.8)$.

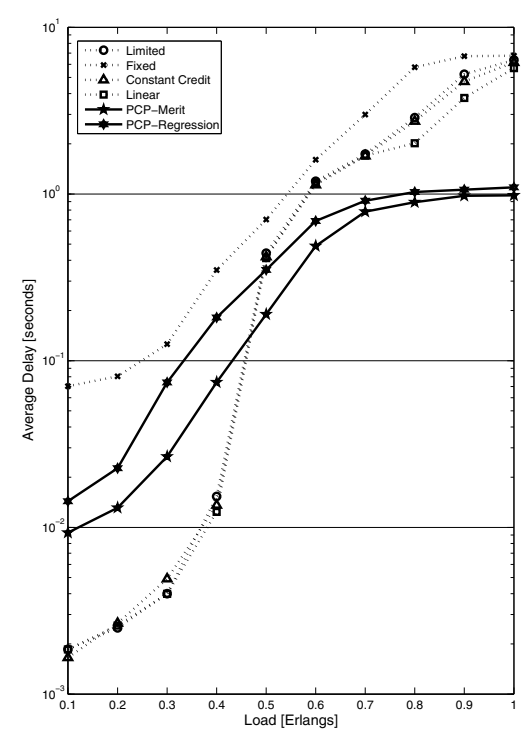

Figure 1.Average delay under long range dependent traffic

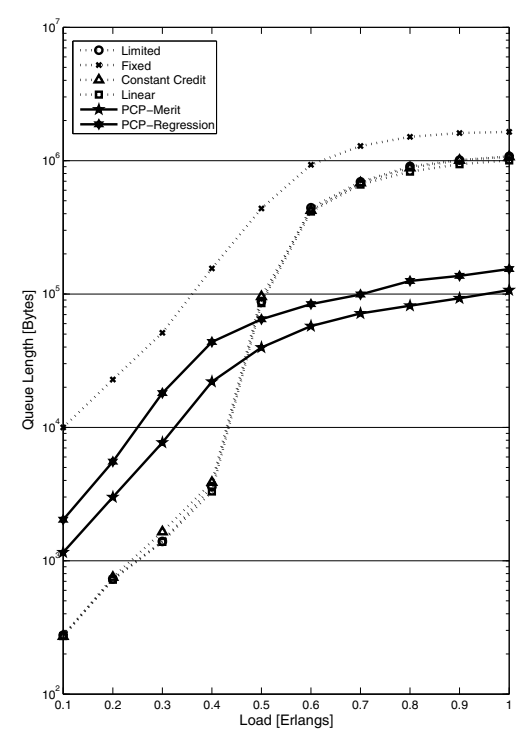

Figure 2. Average queue length under long-range dependent traffic.

As it is seen in Figure 1, at light loads, fixed service has the highest packet delay due to fixed transmission cycle with idle timeslots. PCP-Merit and PCPRegression has significantly lower delay since the average delay, RTT and the buffer growth of the ONUs are taken into account both by individually and collaboratively. Limited, constant credit and linear credit services show performance similar to each other at each load. These triple perform the best at light loads. However, as the traffic offered by the sources increases to moderate and heavy loads, PCP schemes perform significantly better than all of the techniques while the other services lead to the closer delay values to each other. The reason of such a behavior is due to the fact that these services do not provision the network using a dependent scheme among the ONUs while PCP does. Therefore as the offered load gets higher PCP succeeds in delivering a fair polling service to the ONUs by causing an average delay around some 100ms. Besides fairness, the resource utilization is also improved since the average delay per packet is decreased under heavy traffic. It is also seen that PCP-Merit shows better performance than PCP-Regression. 
The results showing that the average queue length of each service discipline are closely related to the results obtained for the average packet delay. The delayed packets force the buffer of the ONU to increase in size. Therefore, it is expected that the behavior of the services due to the average queue length would be similar to the behavior of the results due to the average packet delay. This intuitive analysis is supported by the results shown in Figure 2. At heavy loads, all the other schemes have results close to each other. At light loads, fixed service causes larger queue length since as a result of fixed assignment it assigns insufficient timeslots to the ONUs with heavier buffers. Here, PCP schemes result significantly lower queue lengths as the offered load increases since it reduces the packet delay duration as it is seen in the previous figure.

In the simulation results, it can also be seen that at low loads (less than 0.4 Erlang) PCP-Merit and PCP-Regression leads to higher average delay and average queue length in comparison to limited service, constant credit service, and linear credit service (it still outperforms fixed service). The reason is that PCP schemes attempt to creditize each incoming request based on the RTT, average delay and the buffer growth. However, at low loads, the buffer growth level of the ONUs are so low that an additional credit for the incoming requests may lead to some small idle timeslots which may increase the average delay.

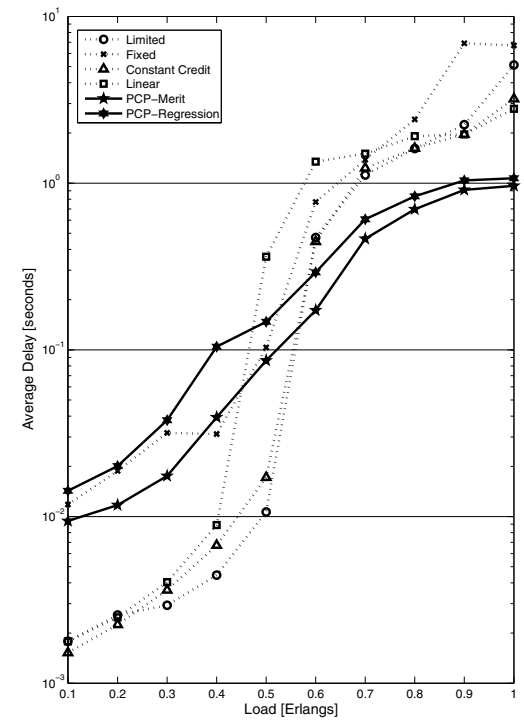

Figure 3.Average delay under Poisson traffic

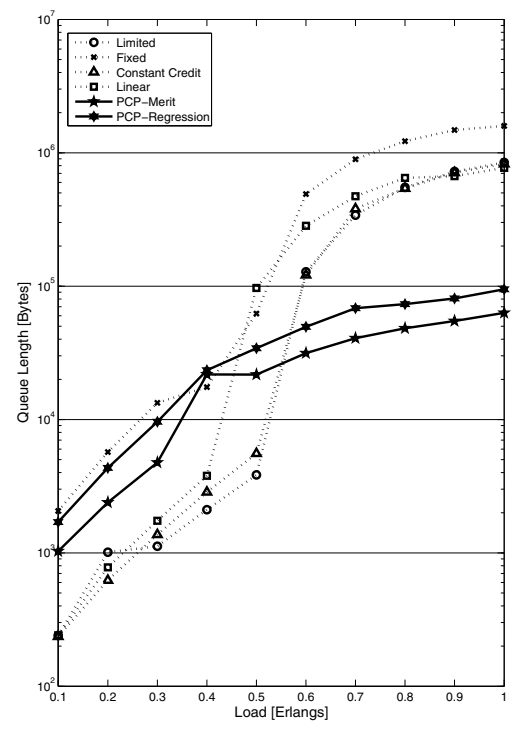

Figure 4. Average queue length under Poisson traffic.

The second set of results is collected under Poisson traffic. The performance behaviors of the services are similar compared to the behavior under long range dependent traffic. However, all of the services lead to slightly lower packet delays 
as shown in Figure 3. The performance improvement of PCP schemes is still significant under Poisson traffic. On the other hand, it has to be mentioned that the numeric values of average packet delays of all schemes are slightly less than the results taken under long range dependent traffic. However, that slight decrease cannot be observed explicitly since these figures are in logarithmic scale.

Based on Figure 4, average queue lengths lead by the services can intuitively be expected to show a slight decrease. However, since the queue length is strongly related to the delayed packets, PCP-Merit and PCP-Regression are expected to lead to a shorter average queue length as the load gets higher. Besides these, since the delay characteristics of the remaining techniques show similarity as the load gets higher, the average queue lengths that are caused by the techniques tend to get closer to each other. The results shown in Figure 3 support these inferences.

The experimental results in this section show that as the EPON traffic gets heavier PCP schemes increase the performance of IPACT protocol by improving the average packet delay and average queue length for each traffic pattern.

\section{Conclusion}

In this paper, we proposed a new bandwidth allocation scheme, Prudent Creditization Polling (PCP) to improve the performance of IPACT in terms of average packet delay and average queue length. PCP is implemented in two different approaches: 1) a fuzzy functional merit based approach (PCP-Merit), and 2) a linear regression based approach (PCP-Regression). PCP adapts the polling cycle time, based on the creditization of the ONUs. The request of an ONU is creditized based on its running average buffer size (or its differential), average delay it is exposed to, and its RTT value which is computed at the previous transmission period. Hence, the algorithm aims to decrease the average packet delay by supplying longer transmission window to the ONUs which are exposed to higher delays and faster running buffer growth. We compare the performance of PCP-Merit and PCP-Regression with the other service schemes (Limited, Fixed, Constant Credit, and Linear Credit) under self-similar and Poisson traffic by simulation. The simulation results show that as the offered load gets higher, PCP leads to a significantly lower average packet delay and average queue length in comparison with the previously proposed schemes.

We are working on the effects of the service schemes on the Hurst parameter value of the traffic going through the system. We also plan to construct an enhanced version of PCP where it pays attention to the QoS requirements of the incoming traffic.

\section{References}

1. Effenberger F. J., Ichibangase H., Yamashita H.: Advances in broadband passive optical networking technologies. IEEE Communications Magazine, Vol. 39, Issue 12, (Dec. 2001) 118-124 
2. Shin D-B., Lee H-S., Lee H-H., Kim D-Y.: An ONU design for an EPON-based access network. Proc. The 9th Asia-Pacific Conference on Communications, APCC, Vol. 3, (Sept. 2003) $1194-1197$

3. Zhongjin W., Yeo D., Xiaodan G., Bin Z., Layhong T., Lam J., Cheng T. H., Walla R.: MPCP design in prototyping optical network unit of ethernet in the first mile. Proc. The Ninth International Conference on Communications Systems, ICCS (Sept. 2004) $121-125$

4. Kramer G., Mukherjee B., Pesavento G.: IPACT a dynamic protocol for an Ethernet PON (EPON). IEEE Communications Magazine, Vol 40, Issue 2, (Feb. 2002) $74-$ 80

5. Wei Q., Chen F.: A high efficient dynamic bandwidth scheme for QoS over EPON system. Proc. International Conference on Communications, Circuits and Systems, Vol. 1, (May 2005) $599-603$

6. Ghani N., Shami N. A., Assi C., A Raja M. Y.: Quality of service in Ethernet passive optical networks. Proc IEEE/Sarnoff Symposium on Advances in Wired and Wireless Communication, (Apr 2004) 161 - 165

7. Assi C. M., Yinghua Y., Sudhir D., Ali M. A.: Dynamic bandwidth allocation for quality-of-service over Ethernet PONs. IEEE Journal on Selected Areas in Communications, Vol 21, Issue 9, (Nov. 2003) $1467-1477$

8. Zhu Y., Ma M., Cheng T. H.: An Urgency Fair Queueing Scheduling to Support Differentiated Services in EPONs. Proc. IEEE Global Telecommunications Conference (GLOBECOM), Vol. 4, (Dec 2005), 1925-1929

9. Hossain A. D, Dorsinville R., Ali M.: Supporting Private Networking Capability in EPON. Proc. IEEE International Conference on Communications (ICC), Vol. 6, (June 2006) 2655-2660

10. Zheng J., Zheng S.: Dynamic Bandwidth Allocation with High Efficiency for EPONs. Proc. IEEE International Conference on Communications (ICC), Vol. 6, (June 2006) 2699-2703

11. Byun H., Nho J., Lim J., Dynamic bandwidth allocation algorithm in ethernet passive optical networks, Electronics Letters, Vol. 39, (June 2003) 1001-1002

12. Miyamoto S., Fuzzy Sets in Information Retrieval and Cluster Analysis, Kluwer Academic Publisher, (1990), Boston

13. Taqqu M. S., Willinger W., Sherman R.: Proof of a fundamental result in selfsimilar traffic modeling. ACM/SIGCOMM Computer Communication Review, Vol. 27, (1997) 5-23 